\title{
The evaluation of B-SAFE and ultrasonographic landmarks in safe orientation during laparoscopic cholecystectomy
}

\author{
Maciej Sebastian¹, Agata Sebastian², Jerzy Rudnicki ${ }^{1}$ \\ ${ }^{1}$ Department of General, Minimally Invasive, and Endocrine Surgery, Wroclaw Medical University, Wroclaw, Poland \\ ${ }^{2}$ Department of Rheumatology and Internal Medicine, Wroclaw Medical University, Wroclaw, Poland
}

Videosurgery Miniinv 2020; 15 (4): 546-552

DOI: https://doi.org/10.5114/wiitm.2020.100972

\begin{abstract}
Introduction: Even though the prevalence of bile duct injury (BDI) is nowadays lower than before and close to the era of open cholecystectomy, there is a strong need to make it even lower. B-SAFE is a group of five visual landmarks that may be used before dissection in the hepatocystic triangle for better orientation around the gallbladder. Another method is laparoscopic ultrasound (LUS), which enables confirmation of structures in the hepatoduodenal ligament and delineation of the safe plane of dissection.

Aim: To evaluate the use of B-SAFE and ultrasonographic landmarks during laparoscopic cholecystectomy in navigation around the gallbladder.

Material and methods: The study group consisted of 158 patients with symptomatic cholecystolithiasis. The methods of intraoperative orientation around the gallbladder attempted in every patient during laparoscopic cholecystectomy included B-SAFE and ultrasonographic landmarks.

Results: The identification rate of ultrasonographic landmarks - the upper border of "Mickey Mouse" sign (MMS) (the equivalent of the Rouviere's sulcus), the bile duct, and the hepatic artery - was significantly higher in patients with body mass index $\geq 30 \mathrm{~kg} / \mathrm{m}^{2}$ and fibrosis and chronic inflammation in the gallbladder neck than B-SAFE. LUS was also significantly more successful in the identification of the bile duct in the whole study group than B-SAFE. There were no significant differences according to the identification of the duodenum. The conversion rate was $2.6 \%$, and we did not observe any BDI.

Conclusions: Visual landmarks defined in B-SAFE are not as reliable as ultrasonographic landmarks; thus, LUS should be taken into consideration in the first place as a method of navigation around the gallbladder.
\end{abstract}

Key words: cholecystolithiasis, laparoscopy, cholecystectomy, ultrasonography, bile ducts.

\section{Introduction}

Laparoscopic cholecystectomy is currently the gold standard treatment for gallstone disease and one of the most common general surgical procedures performed worldwide [1-4]. The generally accepted rule of intraoperative visual identification of the cystic duct and the cystic artery during laparoscopic cholecystectomy is the critical view of safe- ty. Nevertheless, bile duct injury (BDI) still occurs at a declining rate of $0.08-0.3 \%$ reflecting better equipment, increased experience, and moving beyond the "learning curve" [5-10]. The difficulties in reducing the number of BDI below a certain level force the surgeons to develop new methods of intraoperative visual orientation. Among of them are five B-SAFE landmarks (mnemonic): the Bile duct, the Sulcus of Rouviere, the left hepatic Artery pulsation, the um-

\section{Address for correspondence}

Maciej Sebastian MD, PhD, Department of General, Minimally Invasive and Endocrine Surgery, Wroclaw Medical University, Wroclaw,

Poland, phone: +48 607868 096, fax: +48 607868 096, e-mail: mseba@op.pl 
bilical Fissure, and the duodenum (Enteric) used for surgeon orientation around the gallbladder before dissection in the hepatocystic triangle [3, 11, 12]. Another method that may be used for orientation during laparoscopic cholecystectomy before the critical view of safety was obtained is laparoscopic ultrasound (LUS). LUS is non-invasive, non-irradiating, and may be repeated as many times as it is needed with the possibility of differentiation between vascular and avascular structures [13-15].

\section{Aim}

In our study, we are trying to evaluate the use of B-SAFE and ultrasonographic landmarks during laparoscopic cholecystectomy. A comparison of these techniques used in navigation around the gallbladder before dissection in the hepatocystic triangle may help the surgeon to choose the best method to define a safe plane of preparation.

\section{Material and methods}

The study group consisted of 158 patients (98 women and 60 men) operated on between April 2018 and March 2020 in one Department of Surgery. The inclusion criterion for the study was symptomatic cholecystolithiasis, which was characterised by episodes of biliary colic and symptoms of chronic cholecystitis. It included pain that was severe and episodic, located in the epigastrium or the right upper quadrant, and lasted 1 to $5 \mathrm{~h}$. It often woke the patient at night or began after a fatty meal. The commonly associated symptoms were nausea and vomiting. Abdominal ultrasound was performed to confirm the diagnosis. Exclusion criteria were pre- or postoperatively diagnosed cancer of the gallbladder (1 patient was excluded due to postoperatively diagnosed cancer of the gallbladder), preoperative acute cholecystitis, and previous operations in the abdominal cavity. Written informed consent was obtained from all patients before surgery. All procedures followed the ethical standards of the 1964 Declaration of Helsinki and its later amendments, and the study was reviewed and approved by the Ethical Committee of the Medical University (approval number BW-24/2020). Cholecystectomies were performed on an elective basis by three surgeons experienced in LC (> 150 cholecystectomies) and LUS (> 70 examinations). For LUS we used a Toshiba PEF-704 LA laparoscopic probe (frequency $7.0-10 \mathrm{MHz}$ ) and a Toshiba NemioMX SSA-590A diagnostic ultrasound system, all manufactured in Japan. The LUS probe was inserted through an epigastric $10 \mathrm{~mm}$ trocar to obtain the transverse view. The first method of intraoperative orientation during laparoscopic cholecystectomy around the gallbladder was to use the five B-SAFE landmarks. It included the visualisation of any portion of the bile duct, the presence of the sulcus of Rouviere in the form of a "triangle", "hole", or "notch" in the liver parenchyma on the right side of the gallbladder, left hepatic artery pulsation in the form of the pulsation on the left side of the porta hepatis, and the presence of an umbilical fissure and the duodenum (Photo $1 \mathrm{~A}$ ). The presence (or absence) of landmarks was confirmed before any dissection in the hepatocystic triangle after removal of adhesions with the gallbladder and retraction of the gallbladder with the grasper. The second method included the confirmation of three structures of the hepatoduodenal ligament with LUS and colour Doppler function. These were: the bile duct, the proper hepatic artery, and the portal vein, which formed the characteristic MMS (Photos 1 B, 2 A, B). The upper border of MMS was treated in our study as the equivalent of Rouviere's sulcus (Photo 2 B). The presence of enteric structures was also confirmed with LUS (Photo $2 \mathrm{C}$ ). The result of orientation was dissection in the hepatocystic triangle and establishment of the critical view of safety with its three components. It included the clearance of the hepatocystic triangle of all the fibro-fatty and soft alveolar tissue and exposure of the lower third of the cystic plate with only two tubular structures (the cystic duct and the cystic artery) visible entering the gallbladder. The establishment of five B-SAFE landmarks and LUS was attempted routinely in every patient. We set $3 \mathrm{~min}$ as the upper time limit to obtain landmarks. Beyond this time we did not search for the landmarks and stated that they were absent.

\section{Statistical analysis}

Statistical analysis included the Mann-Whitney $U$ test for continuous and Fisher's exact test for binary variables. The level of statistical significance was set at $95 \%(p<0.05)$.

\section{Results}

LUS was significantly more successful in the identification of the upper border of the MMS, the 

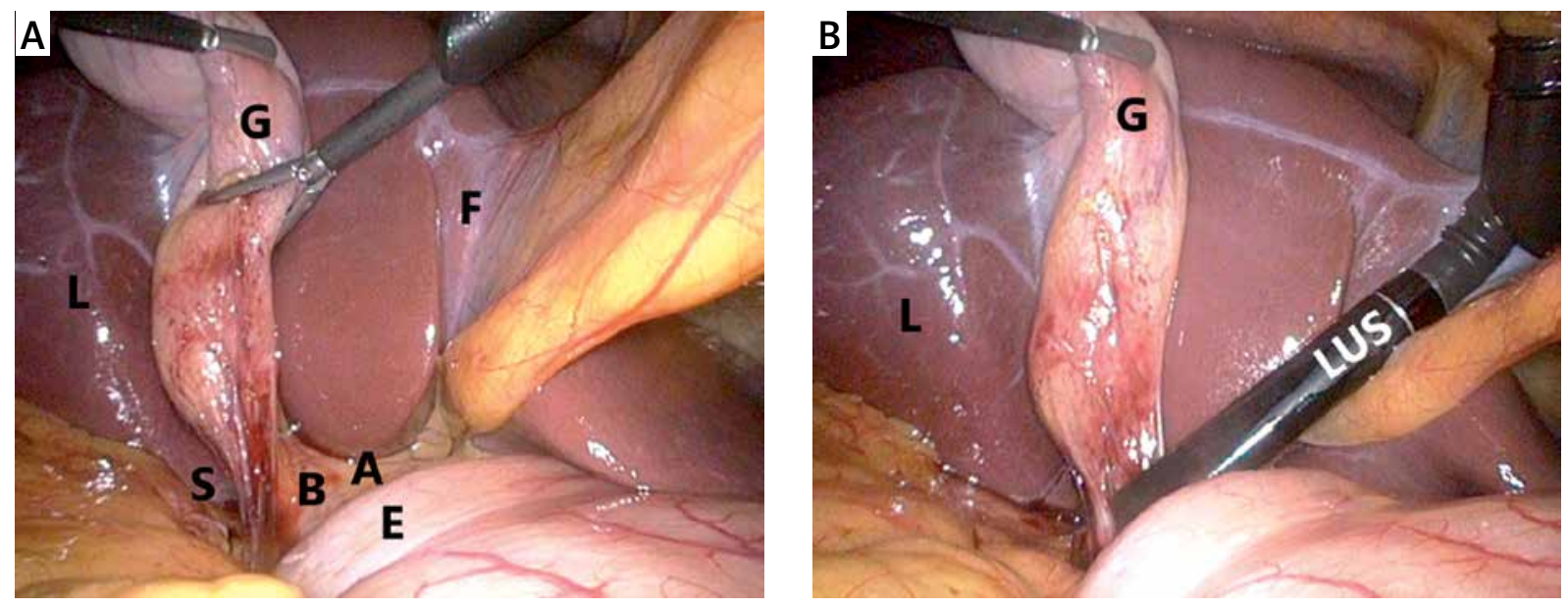

Photo 1. A - B-SAFE landmarks visible during laparoscopic cholecystectomy. B - LUS is used to obtain ultrasonographic landmarks

$A$ - arterial pulsation, B - bile duct, E-duodenum, F-umbilical fissure, $G$ - gallbladder, S - sulcus of Rouviere, L - liver, LUS - laparoscopic ultrasound.
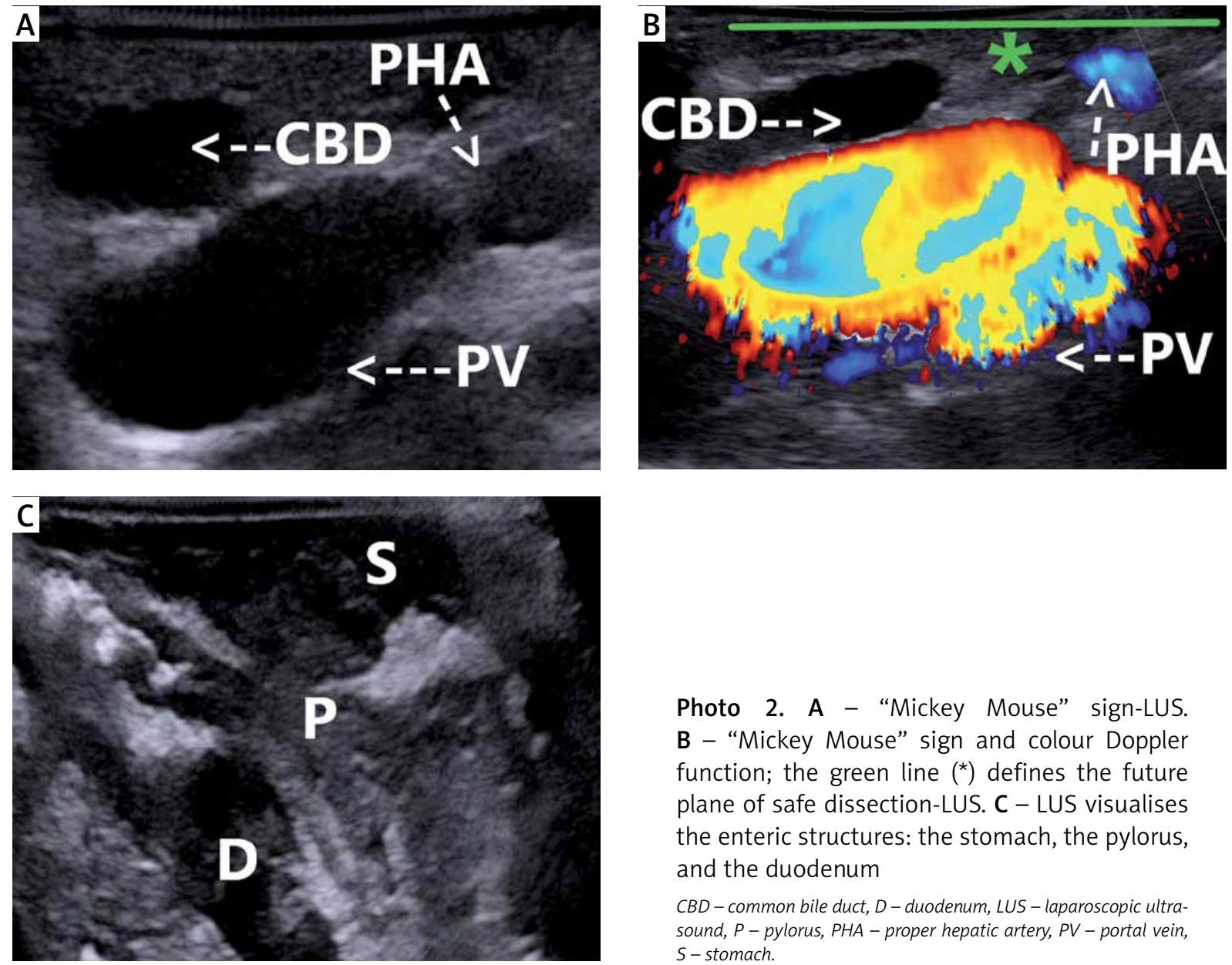

Photo 2. A - "Mickey Mouse" sign-LUS. B - "Mickey Mouse" sign and colour Doppler function; the green line $\left(^{\star}\right)$ defines the future plane of safe dissection-LUS. C - LUS visualises the enteric structures: the stomach, the pylorus, and the duodenum

CBD - common bile duct, D - duodenum, LUS - laparoscopic ultrasound, $P$ - pylorus, PHA - proper hepatic artery, PV - portal vein, $S$-stomach. 
bile duct, and the hepatic artery pulsation in patients with body mass index $(\mathrm{BMI}) \geq 30 \mathrm{~kg} / \mathrm{m}^{2}$ and fibrosis and chronic inflammation in the gallbladder neck than B-SAFE. LUS was also significantly more successful in the identification of the bile duct in the whole study group than B-SAFE. There were no significant differences between LUS and B-SAFE according to the identification of the upper border of the MMS, Rouviere's sulcus, and the arterial pulsation in the whole study group. There were also no significant differences according to the identification of duodenum in the whole study group, in patients with BMI $\geq 30 \mathrm{~kg} / \mathrm{m}^{2}$ and fibrosis and chronic inflammation in the gallbladder neck (Table I). We did not compare the identification of the umbilical fissure because there is no equivalent of this anatomical structure in LUS. The conversion was performed in the early stage of operation in $2(1.3 \%)$ patients. They had only the umbilical fissure identified (B-SAFE) without visualisation of MMS (LUS) due to undissectable fibrous tissue and chronic inflammation in the region of the gallbladder neck. Due to the undissectable fibrous tissue in the region of the hepatocystic, triangle, conversion was performed in the later stage of operation in the next $2(1.3 \%)$ patients. In both patients all four ultrasonographic landmarks were confirmed, from B-SAFE: in 1 patient there were three landmarks, apart from the sulcus of Rouviere and the bile duct, and in the second patient there were three landmarks, apart from the bile duct and the arterial pulsation. There were no significant differences according to the amount of time needed to obtain B-SAFE and LUS landmarks (3.2\% vs. 3.0\% of the mean operating time, respectively) (Table I). We did not observe any BDI.

\section{Discussion}

The main reason for BDI is not the lack of skill, inadequate knowledge, or misjudgement but misperception [16]. The initial false anatomic interpretation or inappropriate orientation before dissection in the hepatocystic triangle may lead to BDI during laparoscopic cholecystectomy $[15,17,18]$. B-SAFE landmarks were developed in response to this problem to help the surgeon in orientation around the gallbladder [3, 12, 19]. Schendel et al. stated that the key landmark to visualise during cholecystectomy is the bile duct, not the gallbladder. Good landmarks in surgery should meet three requirements: they must be present in a high percentage, they must be easy to find and recognise, and their visualisation must be associated with important structures that are going to be dissected [3].

The sulcus of Rouviere is an established landmark for superior/inferior orientation and identification of the plane of the common bile duct; any dissection below the sulcus may result in $\mathrm{BDI}[3,20,21]$. There are three basic forms of this landmark, an open triangle being the most common one with the right portal pedicle mostly present on its floor $[3,21]$. The prevalence of the Rouviere's sulcus is $80-90.6 \%$ (in our study $71 \%$ ), with the group of $10-29 \%$ of patients where it is absent making the B-SAFE method less reliable [3, 22]. Conversely, the upper border of MMS was identifiable in our study in $99 \%$ of patients. Only 2 patients without this landmark were characterised by undissectable advanced chronic inflammation and fibrosis which disabled access to the gallbladder's neck, leading to prompt conversion. Especially in the group of obese patients (BMI $\left.\geq 30 \mathrm{~kg} / \mathrm{m}^{2}\right)$ and in patients with chronic inflammation and fibrosis, LUS enabled significantly better visualisation of the upper border of MMS. Every dissection that is performed over this border is safe and is independent of the traction applied to the gallbladder wall. The landmark moves together with the gallbladder, and its presence can be confirmed in every moment with LUS. Unlike the upper border of MMS, Rouviere's sulcus is a fixed point, and it is possible that during traction or in the case of chronic inflammation and fibrosis the structures of the hepatoduodenal ligament will be located under this landmark.

Regarding the identification of two other structures of the hepatoduodenal ligament, the bile duct and the arterial pulsation, LUS in our study was significantly more successful than B-SAFE, especially in the group of obese patients $\left(B M I \geq 30 \mathrm{~kg} / \mathrm{m}^{2}\right)$ and in patients with chronic inflammation and fibrosis. Arterial identification in B-SAFE used to fix the left/ right position is not direct but we can only assume it from arterial pulsation on the left side of the porta hepatis. The bile duct in obese patients or in case of fibrosis will be obscured through the overlying tissues [3]. LUS bypasses these obstacles because without any additional dissection it enables very precise identification of these structures behind the visible plane. The duodenum is usually not hard to find, and being too close to it means that surgeon is too low and dissection will be close to the common 


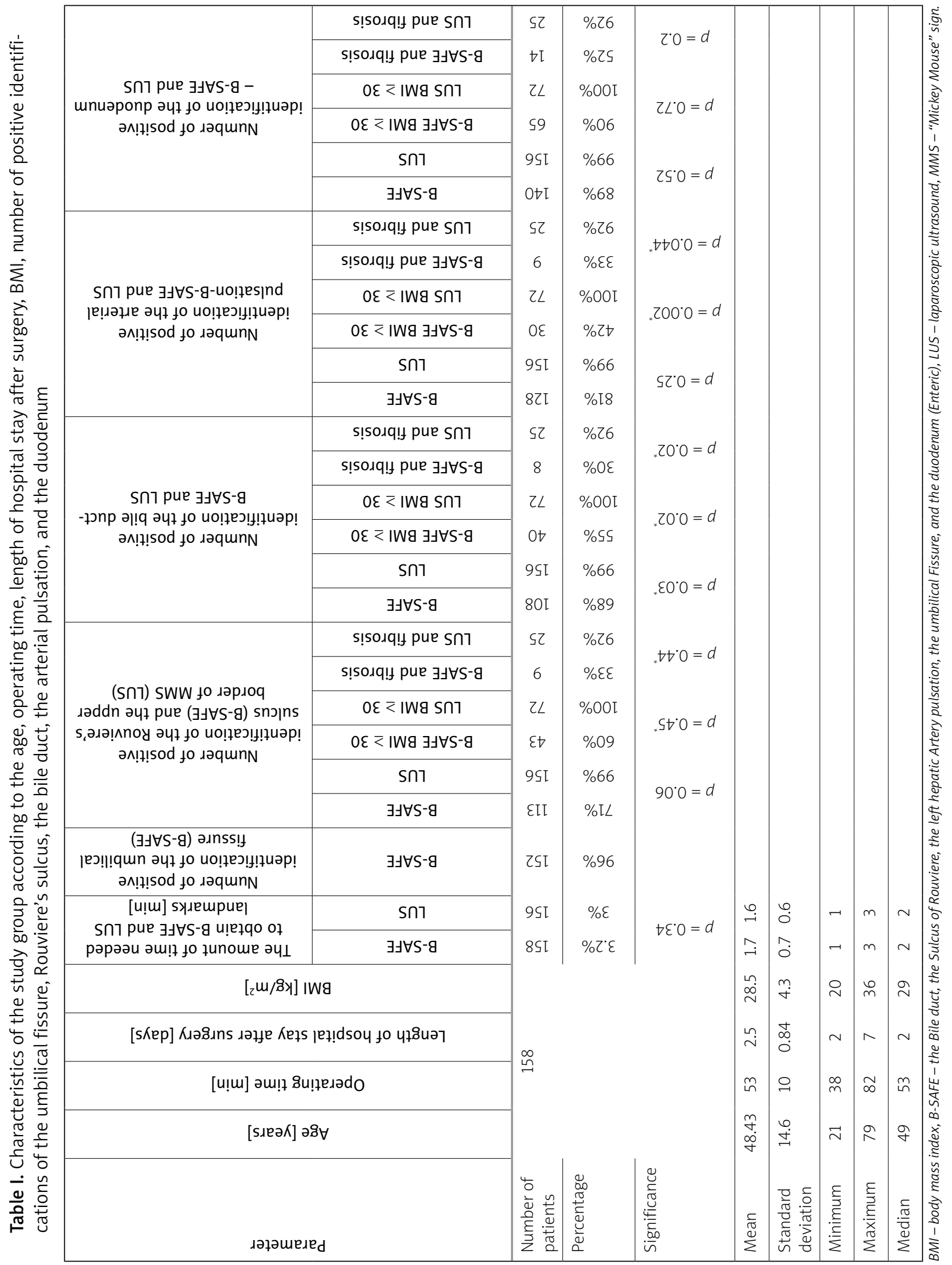


bile duct [3]. In a study by Schendel et al. only $65 \%$ of patients had all four landmarks (in our study 68\%) visualised in B-SAFE. We did not include the umbilical fissure used for left/right orientation, which is located between the left lateral (segments 2 and 3 ) and left medial section (segment 4) where the falciform ligament and ligamentum teres lie, because there is no equivalent of this structure in LUS; the dissection should start to the right of this structure [3]. LUS in our study enabled visualisation of $99 \%$ of ultrasonographic landmarks in the whole study group, in $100 \%$ of patients with $\mathrm{BMI} \geq 30 \mathrm{~kg} / \mathrm{m}^{2}$, and in $92 \%$ of patients with chronic inflammation and fibrosis.

LUS is safe, quick, noninvasive, non-irradiating, and may be performed an infinite number of times whenever it is needed. Intraoperative cholangiography is time-consuming, irradiating, and associated with cystic duct cannulation after dissection in the hepatocystic triangle. Near-infrared fluorescent cholangiography visualises only the bile duct. On the other hand, LUS can be performed before any dissection in the hepatocystic triangle, and it visualises and differentiates the vascular and avascular structures behind the visible plane of future dissection with potential protection against BDI and vasculobiliary injury $[13,15]$. The confirmation of ultrasonographic landmarks during LUS may also play an important role in photographic documentation, which may be used for teaching and medicolegal purposes [18]. In comparison to LUS, which is associated with the use of an expensive laparoscopic ultrasound probe, the advantage of B-SAFE seems to be only the cost-free nature of this technique. With reference to the rate of visualisation and quality of the key landmarks, especially in groups where the risk of $\mathrm{BDI}$ is higher, its efficacy is significantly lower. Therefore, LUS should be primarily recommended for obese patients with chronic inflammation of the gallbladder as a time-efficient and effective method of navigation around the gallbladder. Patients with "preoperative acute cholecystitis" were excluded from the study because we were trying to have as homogenous a group as possible, operated by the same surgeons experienced in LUS, to obtain high-quality of data. The range of acute cholecystitis is very wide, from mild cases to abscesses, necrosis, perforations, and fistulas to the surrounding organs, making comparative statistical analysis more complicated and necessitating a larger group of patients than in our study. Acute cases are operated usually on duties also by surgeons not experienced in LUS or residents, and time-pressure usually discourages the performance of technically demanding examinations. Of course, B-SAFE may be performed in "acute cholecystitis" cases, but we aimed to compare it with LUS, which in such circumstances is limited due to the above-mentioned reasons.

The recent development of artificial intelligence systems used for navigation during laparoscopic cholecystectomy, which are based on the identification of visual landmarks: the cystic duct, the common bile duct, the lower edge of the left medial liver segment, and the Rouviere's sulcus, could also be based on ultrasonographic landmarks, which are more common and reliable [23]. Perhaps in the future the laparoscopic view will be connected with the ultrasound probe for better navigation in the hepatocystic triangle.

The limitation of our study was the relatively small number of patients and its single-centre nature. Further studies including larger groups of participants and preoperative acute cholecystitis cases, in more than one surgical centre, are needed to strengthen our findings and confirm the superiority of LUS over $\mathrm{B}-\mathrm{SAFE}$, especially in protection against BDI.

\section{Conclusions}

Any additional technique that may prevent BDI should be considered among surgeons who perform laparoscopic cholecystectomy. B-SAFE, similarly to the critical view of safety, is only a visual technique, which may be imperfect in some conditions. Conversely, LUS enables effective, functional, and unequivocal confirmation of the safety planes and character of visualised structures associated with the safe laparoscopic cholecystectomy.

\section{Conflict of interest}

The authors declare no conflict of interest.

\section{References}

1. Bogacki P, Krzak J, Gotfryd-Bugajska K, Szura M. Evaluation of the usefulness of the SAGES Safe Cholecystectomy Program from the viewpoint of the European surgeon. Videosurgery Miniinv 2020; 15: 80-6.

2. Agresta F, Campanile FC, Vettoretto N, et al. Laparoscopic cholecystectomy: consensus conference-based guidelines. Langenbecks Arch Surg 2015; 400: 429-53. 
3. Schendel J, Ball C, Dixon E, Sutherland F. Prevalence of anatomic landmarks for orientation during elective laparoscopic cholecystectomies. Surg Endosc 2020; 34: 3508-12.

4. Durowicz S, Kozicki I, Ciesielski A, Tarnowski W. Excision of a part of the bile duct as an iatrogenic injury typical for laparoscopic cholecystectomy - characteristics, treatment and longterm results, based on own material. Videosurgery Miniinv 2020; 15: 70-9.

5. Buddingh KT, Nieuwenhuijs VB, van Buuren L, et al. Intraoperative assessment of biliary anatomy for prevention of bile duct injury: a review of current and future patient safety interventions. Surg Endosc 2011; 25: 2449-61.

6. Strasberg SM, Hertl M, Soper NJ. An analysis of the problem of biliary injury during laparoscopic cholecystectomy. J Am Coll Surg 1995; 180: 101-25.

7. Halbert C, Pagkratis S, Yang J, et al. Beyond the learning curve: incidence of bile duct injuries following laparoscopic cholecystectomy normalize to open in the modern era. Surg Endosc 2016; 30: 2239-43.

8. Mangieri CW, Hendren BP, Strode MA, et al. Bile duct injuries (BDI) in the advanced laparoscopic cholecystectomy era. Surg Endosc 2019; 33: 724-30.

9. Rystedt J, Lindell G, Montgomery A. Bile duct injuries associated with 55,134 cholecystectomies: treatment and outcome from a national perspective. World J Surg 2016; 40: 73-80.

10. Singh R, Brunt LM. Critical view of safety-its feasibilty and efficacy in preventing bile duct injuries. Ann Laparosc Endosc Surg 2018; 8: 2.

11. Gupta V, Jain G. Safe laparoscopic cholecystectomy: adoption of universal culture of safety in cholecystectomy. World J Gastrointest Surg 2019; 11: 62-84.

12. Sutherland F, Dixon E. The importance of cognitive map placement in bile duct injuries. Can J Surg 2017; 60: 424-5.

13. Dili A, Bertrand C. Laparoscopic ultrasonography as an alternative to intraoperative cholangiography during laparoscopic cholecystectomy. World J Gastroenterol 2017; 23: 5438-50.

14. The SAGES safe cholecystectomy program (2014). Available online: https://www.sages.org/safe-cholecystectomy-program/

15. Sebastian M, Rudnicki J. Recommendation for cholecystectomy protocol based on intraoperative ultrasound - a single-centre retrospective case-control study. Videosurgery Miniinv 2020, doi: https://doi.org/10.5114/wiitm.2020.93999.

16. Way LW, Stewart L, Gantert W, et al. Causes and prevention of laparoscopic bile duct injuries: analysis of 252 cases from a human factors and cognitive psychology perspective. Ann Surg 2003; 237: 460-9.

17. Patkin M. Surgical heuristics. ANZ J Surg 2008; 78: 1065-9.

18. Sebastian M, Sebastian A, Rudnicki J. Recommendation for photographic documentation of safe laparoscopic cholecystectomy. World J Surg 2020, doi: 10.1007/s00268-020-05776-9.

19. Gupta, V. Safe laparoscopic cholecystectomy: universal concept, Universal Teaching, Indian Adoption. Indian J Surg 2020, doi: https://doi.org/10.1007/s12262-020-02263-2.

20. Cheruiyot I, Nyaanga F, Kipkorir V, et al. The prevalence of the Rouviere's sulcus: a meta-analysis with implications for laparoscopic cholecystectomy. Clin Anat 2020, doi: 10.1002/ca. 23605.
21. Singh M, Prasad N. The anatomy of Rouviere's sulcus as seen during laparoscopic cholecystectomy: a proposed classification. J Minim Access Surg 2017; 13: 89-95.

22. Lockhart S, Singh-Ranger G. Rouviere's sulcus - aspects of incorporating this valuable sign for laparoscopic cholecystectomy. Asian J Surg 2018; 41: 1-3.

23. Tokuyasu T, Iwashita Y, Matsunobu Y, et al. Development of an artificial intelligence system using deep learning to indicate anatomical landmarks during laparoscopic cholecystectomy. Surg Endosc 2020, doi: 10.1007/s00464-020-07548-X.

Received: 25.10.2020, accepted: 3.11.2020. 